\title{
Supporting information for: Boosting detection of low abundance proteins in thermal proteome profiling experiments by addition of an isobaric trigger channel to TMT multiplexes
}

\author{
Sarah A. Peck Justice†, Neil A. McCracken, José F. Victorinoł, Guihong D. Qi, Aruna B. Wijeratne, \\ Amber L. Mosley* \\ Department of Biochemistry and Molecular Biology, Indiana University School of Medicine, Indianapolis, Indiana \\ 46202, United States \\ $\dagger$ Department of Biology, Taylor University, Upland, Indiana, 46989, United States \\ $\ddagger$ Translational Genomics Research Institute, Phoenix, Arizona, 85005, United States
}

To whom correspondence should be addressed: Tel: (317) 278-2350; Email: almosley@iu.edu

Table of contents:

Figure S1: TMT channels used for each sample

Figure S2: WT protein abundance dot plots

Figure S3: ssu 72-2 protein abundance dot plots

Figure S4: Unique CPF peptide detection

Figure S5: Pti1 phosphorylation detection with CPF trigger channel.

Figure S6: PSMs and ion abundance measurements for CPF subunits in WT technical replicates

Figure S7: Replicate CPF melt curves

Table S1: mTPP data

Table S2: TPP package results, WT no trigger vs WT trigger

Table S3: TPP package results, WT vs. ssu $72-2$

Table S4: Peptide groups for CPF subunits in WT and ssu72-2

Table S5: Changes in Tm and median changes 


\begin{tabular}{|c|c|c|c|c|c|c|c|c|c|c|}
\hline & \begin{tabular}{|l|} 
Trigger or \\
untreated
\end{tabular} & $25^{\circ} \mathrm{C}$ & $35^{\circ} \mathrm{C}$ & $46.2^{\circ} \mathrm{C}$ & $48.8^{\circ} \mathrm{C}$ & $51.2^{\circ} \mathrm{C}$ & $53.2^{\circ} \mathrm{C}$ & $55.2^{\circ} \mathrm{C}$ & $56.5^{\circ} \mathrm{C}$ & $74.9^{\circ} \mathrm{C}$ \\
\hline Trech. rep. No trigger & NA & TMT127N & TMT127C & NA & TMT128N & TMT128C & TMT129N & TMT129C & TMT130N & TMT130C \\
\hline Bio. rep. WT No trigger & TMT126 & TMT127N & TMT127C & TMT128N & TMT128C & TMT129N & TMT129C & TMT130N & TMT130C & TMT131 \\
\hline Bio. rep. WT Trigger p1-3 & TMT126 & TMT127N & TMT127C & TMT128N & TMT128C & TMT129N & TMT129C & TMT130N & TMT130C & TMT131 \\
\hline ssu72-2 Trigger p1-p3 & TMT126 & TMT127N & TMT127C & TMT128N & TMT128C & TMT129N & TMT129C & TMT130N & TMT130C & TMT131 \\
\hline
\end{tabular}

\section{Figure S1: TMT channels used for each sample}

Table denoting which TMT channel was used from a TMTPro (top two rows) or TMT10plex (bottom four rows) for each temperature treated sample. As the same channels were used in each of the three replicates for the WT and ssu72-2 trigger channel experiments, the samples are shown in a single row. For the Bio. Rep. WT and ssu 72-2 No trigger samples, the TMT126 channel was used to label lysate that had not been temperature treated. The remaining TMT126 labeled samples shown are the Pta1-FLAG purification triggers. 

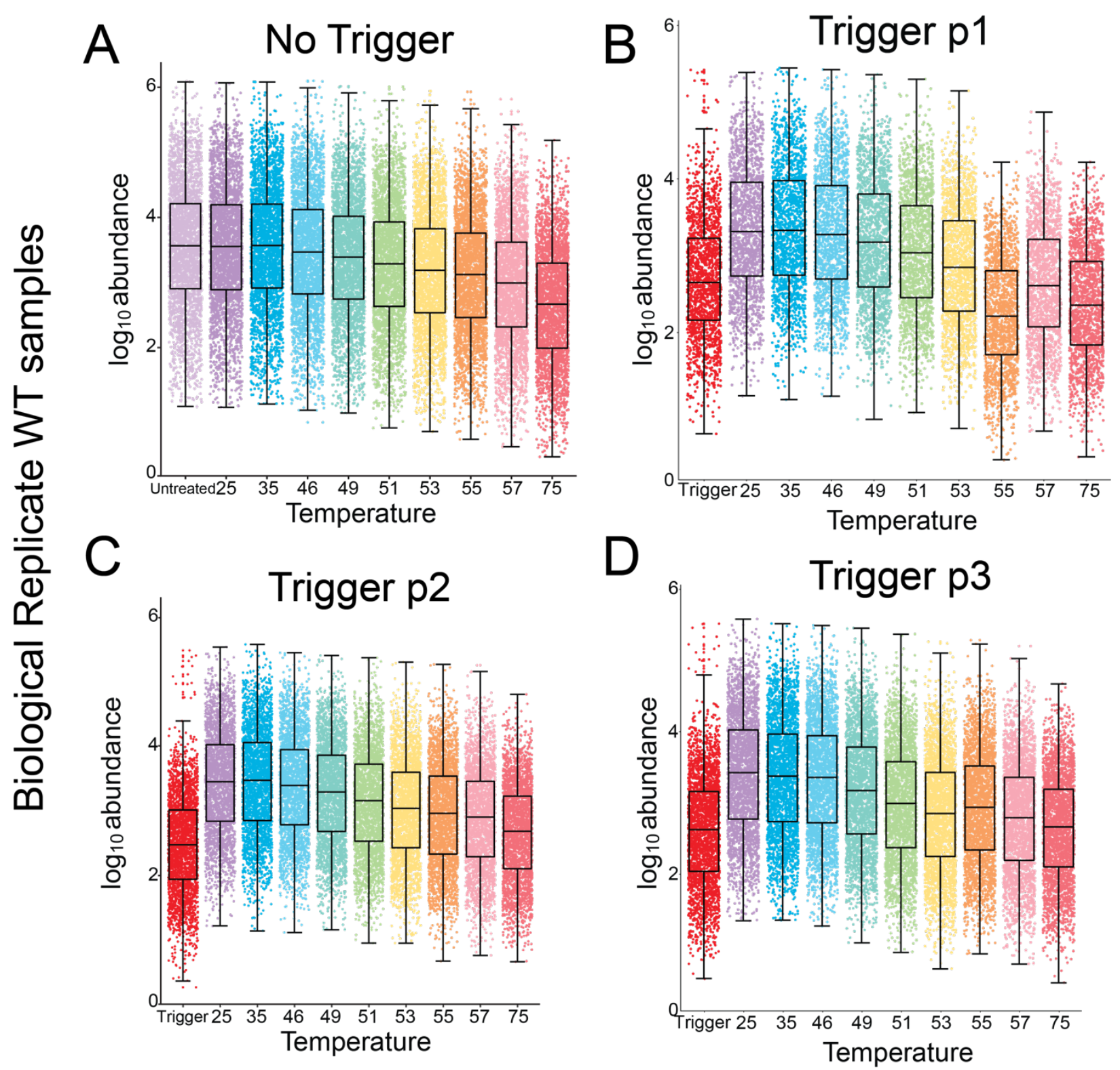

$\mathrm{D}$

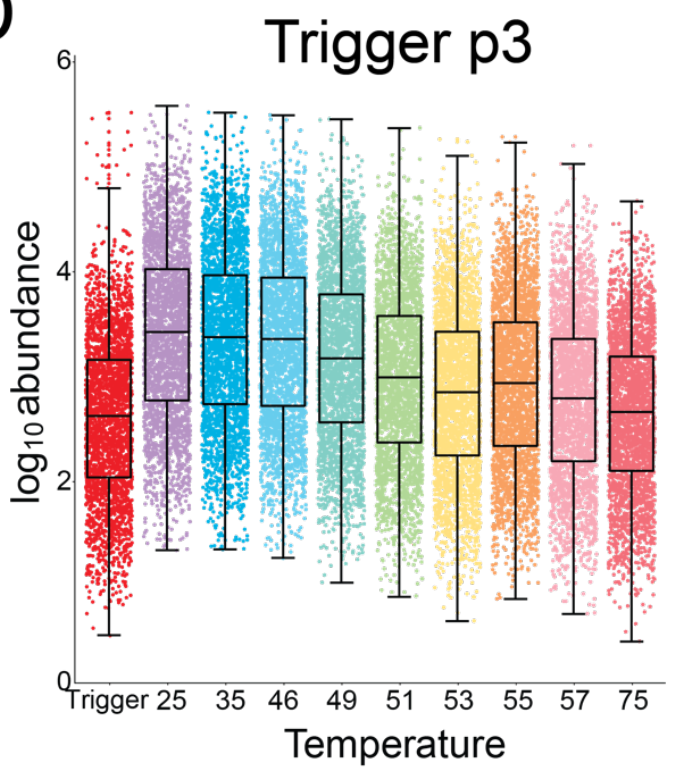

Figure S2. The use of an isobaric trigger channel does not alter mTPP experimental channel abundance values. Dot plots of protein abundance values for each protein detected in WT cells in biological replicates without (A) and with (B-D) the isobaric trigger addition. The same general decrease of protein abundances with increase in temperature treatment is seen across all replicates. 

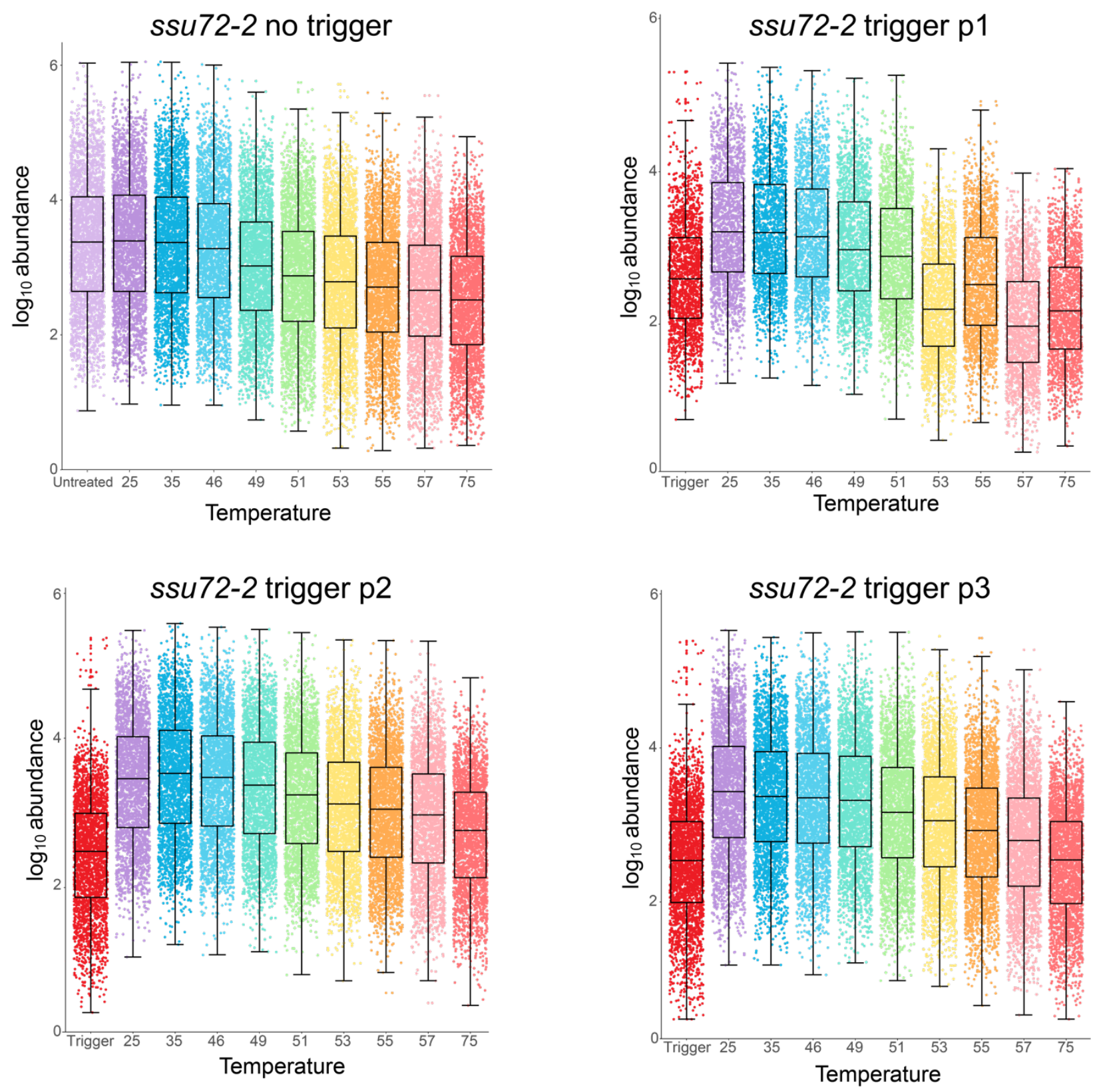

Figure S3: ssu72-2 protein abundance dot plots

Dot plots of protein abundance values for every protein detected in ssu72-2 no trigger and trigger replicates $\mathrm{p} 1, \mathrm{p} 2$, and $\mathrm{p} 3$ 

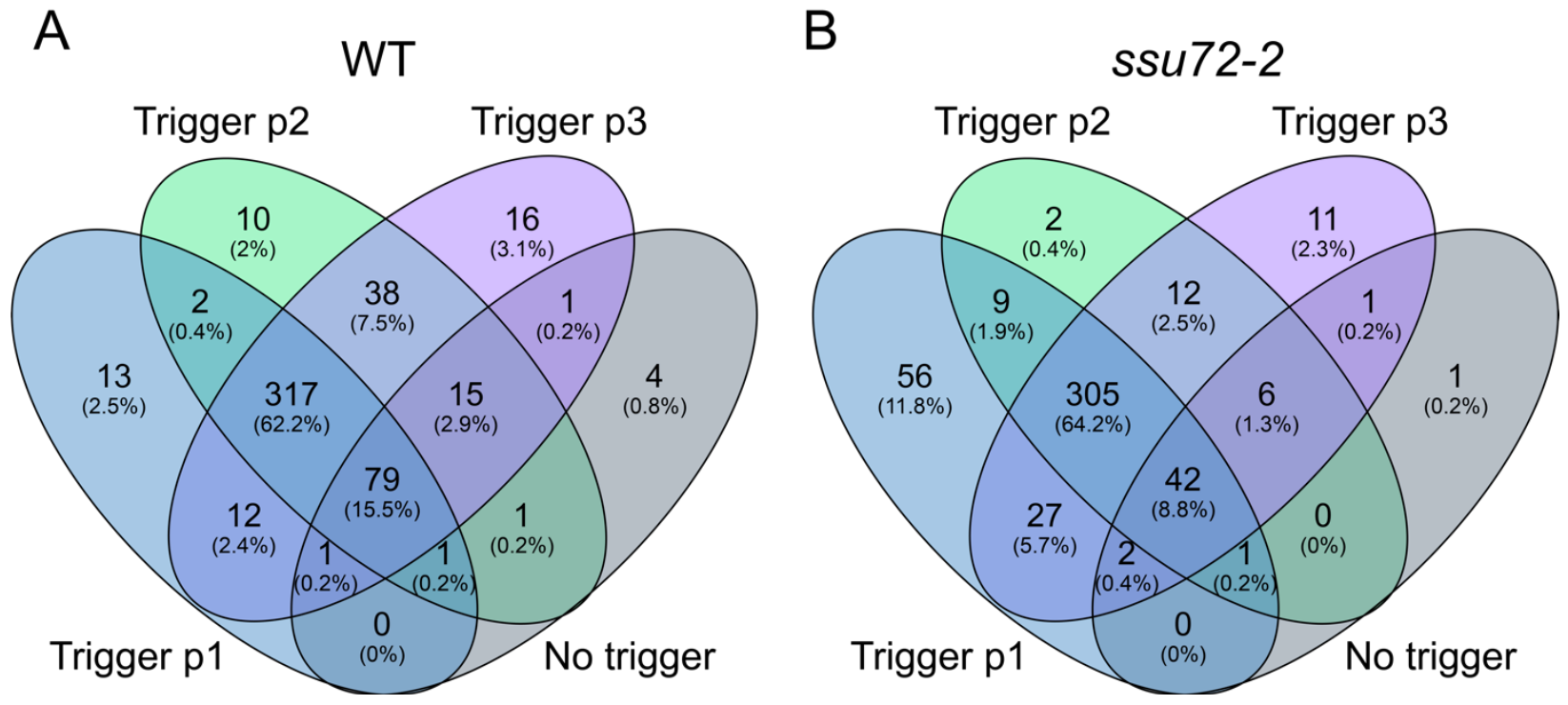

Figure S4: Unique CPF peptide detection

Venn diagrams of the overlap in peptides detected for each of the $14 \mathrm{CPF}$ subunits across each experiment in A) WT and B) ssu 72-2. 


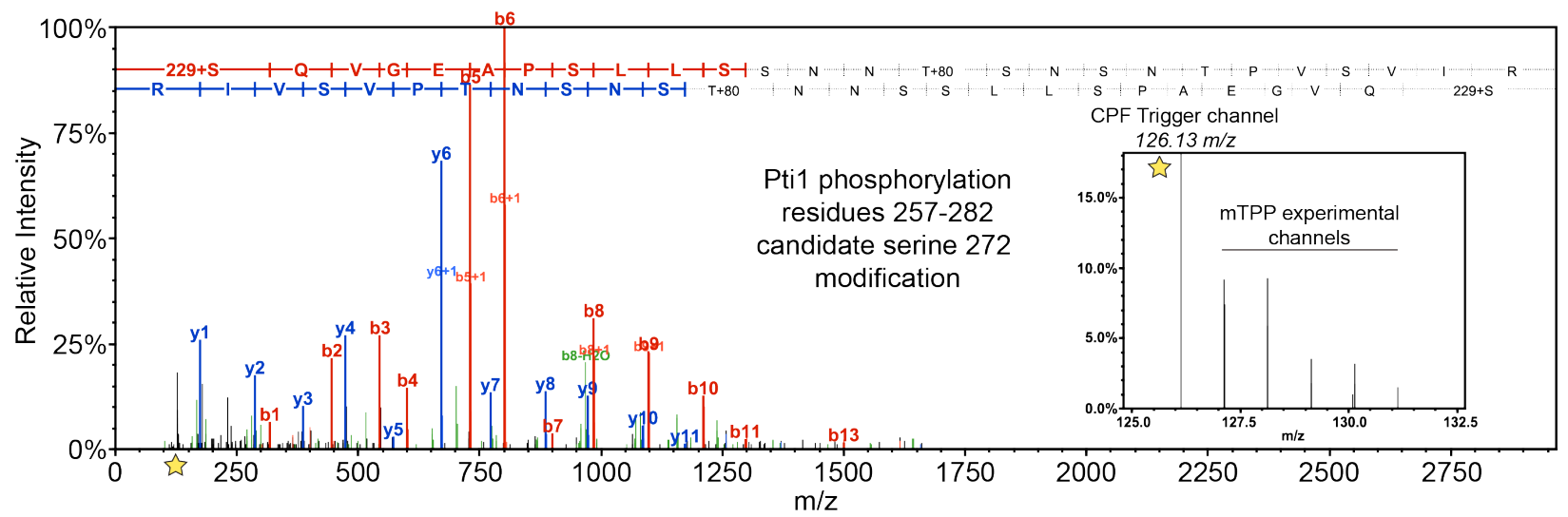

Figure S5. CPF trigger channel allows for detection of specific amino acid modifications. MS2 fragment ion spectrum for Pti1 residues 257 - 282. TMT reporter ions are indicated with a star (left) with a close-up view of the CPF trigger channel signal relative to the mTPP experimental data shown to the right. 

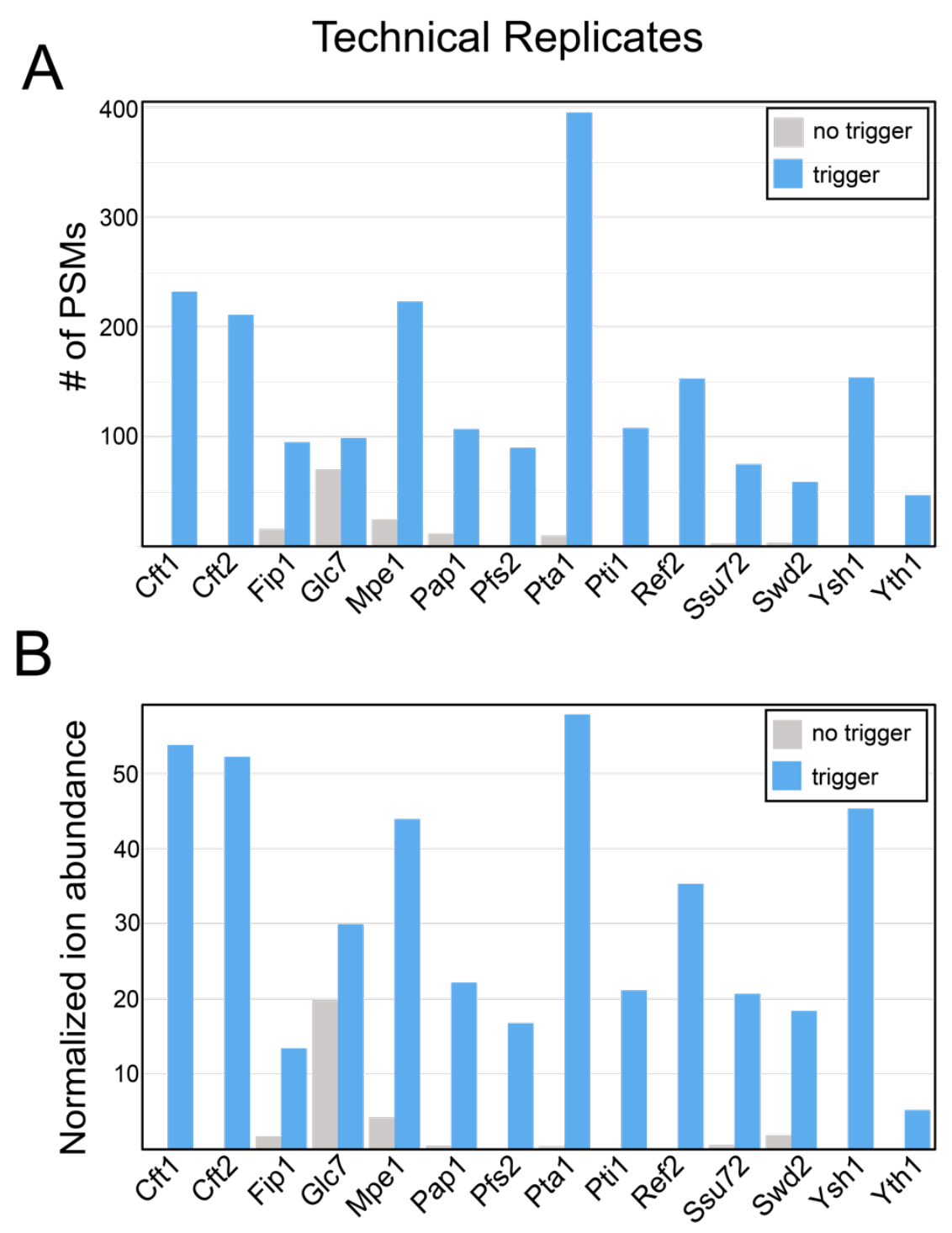

Figure S6: PSMs and ion abundance measurements for CPF subunits in WT technical replicates A) Numbers of PSMs and B) ion abundance normalized to the abundance of Pgk1 for each CPF subunit in WT 
A

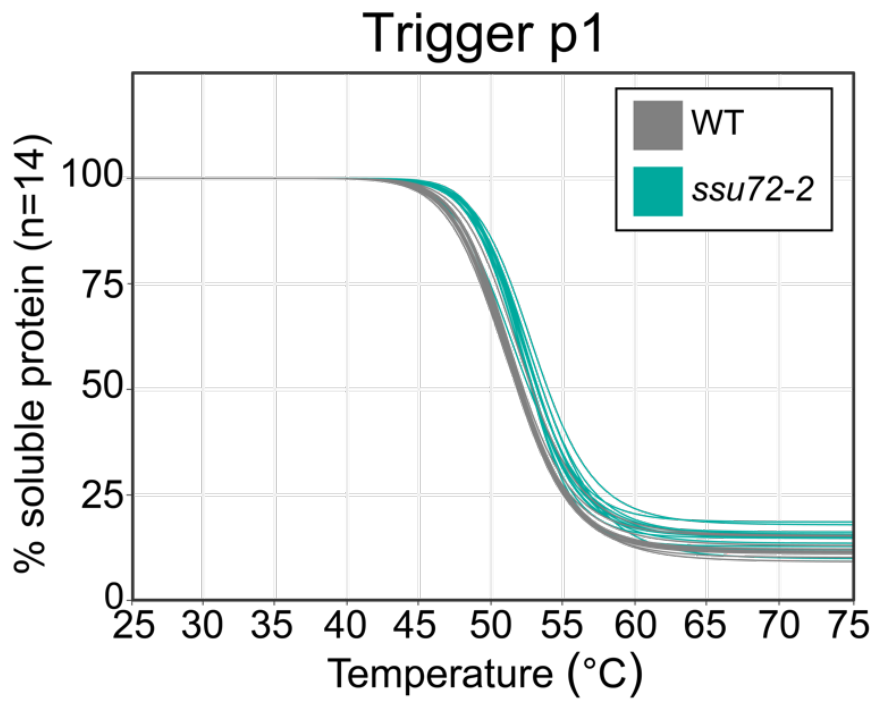

B

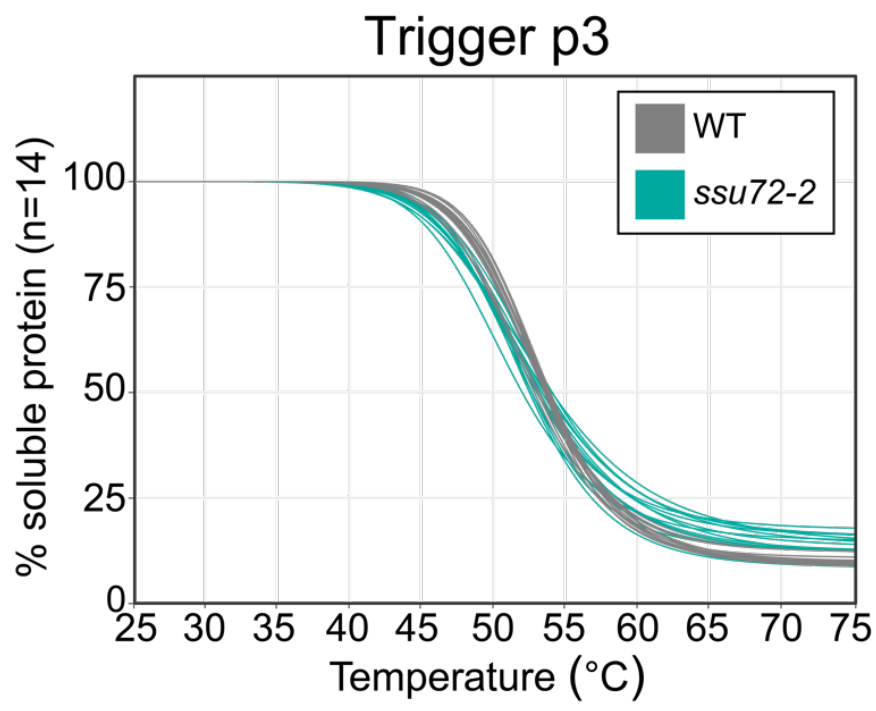

Figure S7: Replicate CPF melt curves

mTPP normalized CPF subunit melt curves. Plots for each of the CPF subunits normalized by the TPP package for trigger $\mathrm{p} 1$ and $\mathrm{p} 3$; trigger $\mathrm{p} 2$ is shown in Figure 5. Curves shown in gray are WT and turquoise are ssu72-2. Each line represents one of the 14 CPF subunits. 


\section{Table S1: mTPP data}

Table of data obtained from mTPP experiments exported from Proteome Discoverer. Each genotype and replicate are provided as a separate sheet within the document (listed below). Provided is the accession number, protein description from Uniprot, Sum PEP score, percent coverage, number of peptides, number of peptide spectral matches (PSMs), number of unique peptides, number of amino acids, molecular weight, raw abundances for each channel, and percent of soluble protein as normalized to the lowest heattreated sample $\left(35^{\circ}\right)$.

Tech rep_No trigger

Tech rep_Trigger

Bio rep_WT_No trigger

Bio rep_WT_Trigger p1

Bio rep_WT_Trigger p2

Bio rep_WT_Trigger p3

ssu72-2_No trigger

ssu72-2_Trigger $\mathrm{p} 1$

ssu 72-2_Trigger $\mathrm{p} 2$

ssu72-2_Trigger $\mathrm{p} 3$

\section{Table S2: TPP package results, WT no trigger vs WT trigger}

Output results provided from the TPP package of the comparing WT no trigger vs WT trigger in technical (sheet labeled Techreps_WT_Notriggervstrigger) and biological replicates (sheet labeled

Bioreps_WT_Notriggervstrigger). Input data provided in Table 1. Data includes the following information for each protein: normalized relative fold changes for each channel, estimated melt temperature, and the slope, plateau, and $\mathrm{r}$ for the protein melt curve.

Table S3: TPP package results, WT vs. ssu72-2

Output results provided from the TPP package comparing WT vs. ssu72-2 in one no trigger biological replicate (data sheet labeled No Trigger) and three trigger biological replicates (data sheets labeled Trigger p1-3). Input data provided in Table 1. Data includes the following information for each protein: normalized relative fold changes for each channel, estimated melt temperature, and the slope, plateau, and $\mathrm{r}^{2}$ for the protein melt curve.

\section{Table S4: Peptide groups for CPF subunits in WT and ssu72-2}

Table of unique peptide groups for each of the CPF subunits in WT and ssu72-2 exported from Proteome Discoverer. Each genotype and replicate are provided as a separate sheet within the document. Provided at the protein level is the accession number, protein description from Uniprot, percent coverage, number of peptides, number of PSMs, number of unique peptides, number of amino acids, molecular weight, protein $\mathrm{pI}$, number of razor peptides, and raw abundances for each channel. For each peptide, data includes the peptide sequence, modifications, number of protein groups, number of proteins, number of PSMs, Uniprot accession for the master protein, the position within the master protein, modifications within the master protein, number of missed cleavages, and raw peptide abundances for each channel.

\section{Table S5: Changes in Tm and median changes}

Table of change in $\mathrm{T}_{\mathrm{m}}$ (calculated from the results in Table 3) and median change in $\mathrm{T}_{\mathrm{m}}$ in ssu72-2 vs WT across the three trigger biological replicates. $\mathrm{T}_{\mathrm{m}}$ values for $s s u 72-2$ were subtracted from WT to get changes in $\mathrm{T}_{\mathrm{m}}$. Median values were calculated for each protein that was quantified and was given a $\mathrm{T}_{\mathrm{m}}$ and $>0.9 \mathrm{r}$ by the TPP package in at least two replicates. 